\title{
Feature issue of digital holography and 3D imaging (DH) introduction
}

\author{
Yoshio Hayasaki, ${ }^{1, *}$ Changhe Zhou, ${ }^{2}$ Gabriel Popescu, ${ }^{3}$ and Levent Onural ${ }^{4}$ \\ ${ }^{1}$ Center for Optical Research and Education (CORE), Utsunomiya University 7-1-2 Yoto, Utsunomiya 321-8585, \\ Japan \\ ${ }^{2}$ Shanghai Institute of Optics and Fine Mechanics (SIOM), Chinese Academy of Sciences, P.O Box 800-211, \\ Shanghai, 201800, China \\ chazhou@mail.shcnc.ac.cn \\ ${ }^{3}$ Department of Electrical and Computer Engineering \& Bioengineering, University of Illinois at Urbana- \\ Champaign, 405 North Mathews Avenue, Urbana, IL 61801, USA \\ gpopescu@illinois.edu \\ ${ }^{4}$ Department of Electrical and Electronics Engineering, Bilkent University, TR-06800 Ankara, Turkey \\ onural@bilkent.edu.tr \\ *hayasaki@opt.utsunomiya-u.ac.jp
}

\begin{abstract}
The OSA Topical Meeting "Digital Holography and 3D Imaging (DH)," was held in Seattle, Washington, July 13-17, 2014. Feature issues based on the DH meeting series have been released by Applied Optics (AO) since 2007. This year Optics Express (OE) and AO jointly decided to have one such feature issue in each journal. The DH meeting will continue in the future, as expected, and the next meeting is scheduled to be held on 24-28 May 2015, in Shanghai Institute of Optics and Fine Mechanics, Shanghai, China.

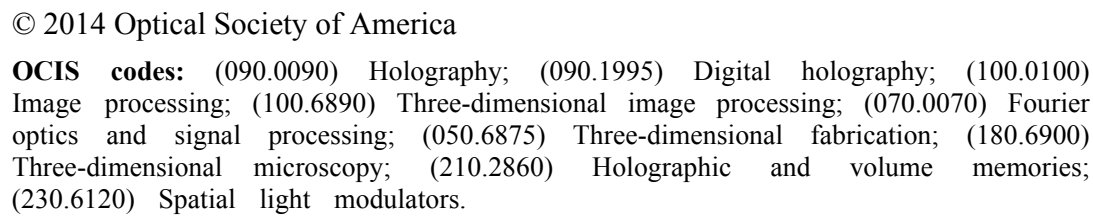

This issue is a follow up of the OSA Topical Meeting "Digital Holography and 3D Imaging (DH)," which was held in Seattle, Washington, July 13-17, 2014. The Topical Meeting provides a forum for science, technology, and applications of the topics such as threedimensional (3D) imaging and display systems, digital holography and holographic microscopy, computer-generated holograms, holographic lithography, holographic remote sensing, biomedical imaging. The meeting has been growing, especially in the number of contributions in applications, and aiming further recognition from other research communities.

The feature issues based on the digital holography and 3D imaging have been released by Applied Optics (AO), since 2007. This year Optics Express (OE) and AO jointly decided to have one such feature issue in each journal as a response to a growing number of new findings on optical phenomena, new developments of methods and techniques, theoretical and experimental analysis of systems, and important improvements.

As we know, both journals have different kinds of characters. Scientific and technology innovations are the main factors for publications to either one of the journals. As for AO, we encouraged authors to give full and in-depth descriptions of the concepts, theory, mathematical models, experimental systems, and experimental results so that a reader can reproduce the results published in the paper. As for OE, we encourage the authors to emphasize the innovations rather than in-depth descriptions. Naturally, we also encourage the use of video multimedia files for authors to demonstrate their innovative works since such supporting material is especially effective in imaging and display fields. 
Thirteen papers are published in this $\mathrm{OE}$ special issue. The total number papers in the two special issues including the one in AO will be more than thirty; we do not know the exact number, at present, since the review process for the AO papers is continuing. They papers cover a large range of topics, reflecting the rapidly expanding techniques and applications of digital holography and 3D imaging. The DH meeting has been going on for eight years, with a growing strength, and the next meeting is scheduled to be held on 24-28 May 2015, at Shanghai Institute of Optics and Fine Mechanics, Shanghai, China. We thank and applaud all the authors in this feature issue as well as the participants in the DH meeting for being a part of this exciting event. 